\title{
Breaking down the barriers between econophysics and financial economics
}

\author{
Franck Jovanovic and Christophe Schinckus \\ School of Management - University of Leicester
}

\begin{abstract}
This article highlights the current misunderstanding between economists and econophysicists by adopting the financial economists' viewpoint in order to explain why the works developed by econophysicists are not recognized in finance. Because both communities do not share the same scientific culture, and for the other reasons developed in the article, economists often consider econophysics as a strictly empirical field without theoretical justification. This paper shows the opposite; it also tries to facilitate the dialogue between econophysicists who often do not explain in details their theoretical roots and financial economists who are not familiar with statistical physics. Beyond this clarification, this paper also allows to identify what remains to be done for econophysicists to contribute significantly to financial economics: 1) development of a common frameworklvocabulary in order to better compare and integrate the two approaches; 2) development of generative models explaining the emergence of power laws; and 3 ) development of statistical tests for the identification of such statistical regularities.
\end{abstract}

JEL classification: G1, N2, B4, B5, B26

Keywords: Statistical physics applied to finance; Power laws; Interdisciplinarity; econophysics; financial economics and econophysics

\section{Introduction}

Econophysics is a recent field that dates back to the 1990s; it applies theories/methods developed by physicists and associated with the physics of complex systems in order to study complex (nonlinear) problems in economics (Daniel \& Sornette, 2010; Jovanovic \& Schinckus, 2013; Săvoiu \& Andronache, 2013). Because physics is a science dealing with a great number of natural issues (matter, energy, light etc.), it potentially offers a wide variety of conceptual tools for studying economic phenomena. Based on the success of the first works in econophysics dedicated to the statistical characterization of fat-tails in financial distributions, the existing literature often associates econophysics with statistical physics applied to finance. Although econophysics cannot methodologically be reduced to this application ${ }^{1}$, this area of knowledge contributed to the crystallization and to the institutionalisation of econophysics (Gingras \& Schinckus, 2012).

Since the birth of econophysics, a huge literature has been published and many results have been provided in finance (Bouchaud et al., 2002; Gabaix, 2009; Lux,

\footnotetext{
${ }^{1}$ The application of statistical physics to economics also deals with corporate revenue (Okuyama et al., 1999), the emergence of money (Shinohara \& Gunji, 2001), or global demand (Donangelo \& Sneppen, 2000).
} 
2009; McCauley, 2009; McCauley et al., 2007; Potters \& Bouchaud, 2003; Sornette, 2014). However, in spite of the numerous publications and of the econophysicists' conviction about their potential contributions to finance, this approach seems to have great difficulties for convincing financial economists. While numerous explanations have been provided by econophysicists for explaining the relatively 'closed' attitude of financial economists (Bouchaud, 2002; Gallegati et al., 2006b; McCauley, 2004, 2006; Sornette, 2014), these explanations refer most of the time to disciplinary reactions rather than theoretical and methodological investigations for solving the problem. Actually, these disciplinary differences can be very informative in the understanding of the situation because part of the gap between the two disciplines, which underlines many debates that have emerged between physicists and financial economists (Ball, 2006; Durlauf, 2005; Gallegati et al., 2006a; Keen, 2003; LeBaron, 2001; McCauley, 2006; Stanley \& Plerou, 2001). In that perspective, statistical physics applied to finance is an interesting area of investigation for one who wants to understand the major differences between these two communities and to go forward the current limits.

This article aims at analysing the main origins of these difficulties in order to contribute to the development of theoretical and methodological bridges between these two disciplines. In this context, this paper studies on the one hand, the reasons for why a lot of financial economists are reluctant to the application of statistical physics to finance, and on the other hand, the possible paths to pass over this situation. Roughly speaking, three arguments are usually mentioned by economists: 1) econophysics would be a data-driven field without theoretical foundations; 2) this field cannot really contribute to the existing theory in finance; and 3) the theoretical framework used in financial economics is not enough considered by econophysicists. This article deals with these three arguments by refuting the first one and discussing the two latter.

The following section will illustrate the heart of the problem between statistical physicists and financial economists by presenting the role played by power laws in the understanding of financial markets. This conceptual introduction will lead us to clarify the theoretical roots of these laws defining the major theoretical foundations of econophysics. Afterwards we will study the major reasons for why this theoretical framework is not currently accepted in finance. We will conclude this paper by discussing what remains to be done for a future integrated perspective between econophysics and financial economics.

\section{The "dialogue of deaf" between econophysicists and financial economists}

Among the branches of physics that can be used for studying financial reality, one is called statistical physics. This latter is a sub-field of physics dealing with statistics and probability theory whose aim is to characterize the properties of matter in aggregate. Given the statistical and probabilistic foundations of finance (Davis \& Etheridge, 2006; Jovanovic, 2008) and the increasing number of financial data (Jovanovic \& Schinckus, 2016), statistical physics appears to be an appropriate branch for studying financial markets because this area of knowledge studies the dynamics of complex systems composed by high number of micro-components (Rickles, 2008). One important discovery of statistical physics is that such as systems can have a macroscopic behaviour with a temporal/spatial scaling- 
invariance property in line with what one can observe at the critical point of a phase transition. As we will explain in details, this observation is called "the self-organized criticality" and it often statistically describes through a power law, which is one of the major theoretical concepts of econophysics.

Although financial economists are familiar with power laws (Gabaix, 2009; Lux, 2009), these patterns did not arouse enthusiasm in economics/finance research. The most obvious reason is that this framework has statistical properties that are not compatible with traditional statistical tests (based on the central limit theorem), and with the financial mainstream founded on a (improved) Gaussian framework. Actually the latter has a historical importance in finance since it played a crucial role in the "scientifisation" of the emergence of finance in the 1960s (Davis \& Etheridge, 2006; Jovanovic, 2008; Jovanovic \& Schinckus, 2016). Consequently, in order to stay into the Gaussian borders by integrating extreme variations (which is one major characteristic of power laws) financial economists have developed alternative approaches: jump processes in the 1970s (Cont \& Tankov, 2004; Merton, 1976) and ARCH class of models in the 1980s (R. F. Engle, 1982; Francq \& Zakoian, 2010; Pagan, 1996). Beyond these considerations related to the importance of the Gaussian framework, power laws also have some drawbacks since their identification and their characterization still generate a lot of debates among scientists. In 2005, for instance, Michael Mitzenmacher published a seminal paper underlying the difficulties in the use of these statistical tools. He explained that empirical results are only a part of the power laws issue because the real challenge is to explain the emergence of these macro-regularities. According to him, generative models explaining the emergence of power laws and quantitative tests to identify these laws are a necessary step in their broad use in science (Mitzenmacher, 2005, p. 526).

The same year, the economist Steven Durlauf (2005) called into question the strength of the empirical evidence for power laws in economic data - in the same vein, Newman (2005) showed that this situation is shared in many other fields. Although Durlauf was one of the defender of complexity in social sciences (including economics) he criticized some works that applied complexity in these sciences whilst completely ignoring the economic methodologyltheory. His critic, which is shared by numerous financial economists and economists, was based on the limits emphasized by Mitzenmacher : the lack of quantitative tests for identifying power laws and the lack of generative models explaining their emergence. These two "lacks" have largely contributed to the maintenance of the Gaussian framework (which has been adapted to extreme variations) by financial economists. These lacks also have strongly supported the questionings of financial economists about the potential contribution of econophysics to their field. Moreover, these calls into question have been reinforced by the rhetoric of some econophysicists who have ignored the economics literature by presenting their results as completely news while they were not always (Lux, 2009) ${ }^{2}$. We will discuss this claim in our last section.

\footnotetext{
2 "One often finds [in the literature from econophysics] a scolding of the carefully maintained straw man image of traditional finance. In particular, ignoring decades of work in dozens of finance journals, it is often claimed that "economists believe that the probability distribution of stock returns is a Gaussian", claim that can easily be refuted by a random consultation of any of the learned journals of this field [...] some of the early econophysics papers even gave the impression that what they attempted was a first quantitative analysis of financial time series ever" (Lux, 2009, p. 230).
} 
In conclusion, the current situation reflects a "dialogue of deaf" between econophysicists and financial economists. The following sections will investigate the reasons of this difficult dialogue between economists and econophysicists. This investigation will lead us to explain the theoretical framework implicitly used by econophysicists when they referred to power laws. This 'translation' (for economists) of the major theoretical framework used in statistical physics is the first contribution of this article because, in their papers, econophysicists usually do not remind these theoretical points (simply because they are well-known by all physicists). For their part, economists, who know few about statistical physics, tend to consider econophysics as a strictly empirical field without any theoretical justification. The next sections will show the opposite by trying to facilitate the dialogue between the two communities.

\section{Statistical physics goes beyond its borders}

For most economists, including financial economists, it is not clear why econophysicists find so obvious the idea to import models lmethods from statistical physics for studying financial markets (or other areas of economics). The influence of physics on economics is nothing new (Ingrao \& Giorgio, 1990; Le Gall, 2002; Ménard, 1981; Mirowski, 1989b; Schabas, 1990) and finance has also been subject to the influence of physics (Jovanovic, 2006a, 2006b; Jovanovic \& Schinckus, 2013, 2016; Sornette, 2014). However, despite these links and the variety of physical tools for studying economic/financial phenomena, econophysics is fundamentally a new approach, very different from the previous experiences. Indeed, its practitioners are not economists taking their inspiration from the works in physics to develop their discipline as it was the case in the history of economics. This time, it is physicists that are going beyond the boundaries of their discipline by using their methods and models to investigate various problems usually studied by social sciences - from this perspective econophysics is really new. This movement outside physics is rooted in changes that occurred in this discipline during the 1970s.

\subsection{Statistical physics' golden age}

A turning point in the recent history of physics that took place in the 1970s was the realization that a connection can be made between the theories of statistical physics and particle physics. Statistical physics' main purpose is to explain the macroscopic behaviour of a system and its evolution, in terms of physical laws governing the motion of the microscopic constituents (atoms, electrons, ions, etc.) that make it up. Statistical physics distinguishes itself from other fields of physics by its methodology based on statistics, a consequence of the enormous number of variables on which statistical physicists have to work. As Fitzpatrick (2012) explains, in areas of physics other than thermodynamics, physicists are able to formulate some exact, or nearly exact, set of equations - resulting from physical laws and theories - which govern the system under investigation. Therefore, they are able to analyse the system by solving these equations, either exactly or approximately. In thermodynamics, physicists have no problem in formulating the governing equations and writing down the exact laws of motion, including all the inter-atomic forces. Their problem is the 
gigantic number of variables - as many as Avogadro's number, $6 \times 10^{23}-$ and therefore the gigantic number of equations of motion that have to be resolved. This number makes study of the system unworkable, even for a computer. The subject is so difficult that physicists "are forced to adopt a radically different approach to that employed in other areas of physics" (Fitzpatrick, 2012, p. 4).

Fortunately, they are not interested in knowing the position and velocity of each individual particle in the system at any time. Instead, they want to know the properties of the system (volume, temperature, etc.) at a given moment. Therefore, the number of pieces of information they require is minuscule in comparison with the number of pieces of information that would be needed to completely specify the internal motion. Moreover, the quantities, which physicists are interested in, do not depend on the motions of individual particles, but rather on the average motions of all the particles in the system. In other words, these quantities depend on the statistical properties of particle motion. Moreover, the gigantic quantity of data makes possible to use most statistical laws or theorems, which are generally based on asymptotic calculus. The method used in statistical physics is thus essentially dictated by the complexity of the systems due to the enormous numbers of constituents. It leads statistical physicists to start with statistical information about the motions of the constituents, and then to deduce some other properties of the system from a statistical treatment of the governing equations. The turning point that occurred in the 1970s is a direct result of this problematic of extremely voluminous data.

Statistical physicists work on the bridge between microscopic level composed by a high number of components and the phenomenological (macro) level of physical systems. In 1982, the physicist Kenneth Wilson received the Nobel Prize for his contribution to such connection. In a sense, his work represents a new approach to critical phenomena that emerged in the 1960s, linking these phenomena with scaling laws (Hughes, 1999, p. 111). Specifically, Wilson was awarded the prize for having developed the renormalization group theory for critical phenomena in connection with phase transitions. A phase transition is a transformation of a state of a system to another state due to the gradual change of an external variable. This transformation can be likened to the passage from one equilibrium to another. When this passage occurs in a continuous way, it passes through a critical point at which neither of the two states is realised. This is a kind of non-state with no real difference between the two states of the phenomenon and for which the system appears the same at all scales. This property is called "scale invariance" meaning that no matter how closely you look, you see the same thing.

Since the 1970s, critical phenomena, which are one of the toughest problems in physics, have captured the attention of physicists due to several important conceptual advances in scaling, universality, and renormalization on the one hand, and to the very interesting properties that define them on the other hand. Among these properties, the fact that the occurrence of their critical points can be characterized by a power law deserved special attention, because this law is a key element in econophysics' literature. During the years 1975-80 statistical physics was blossoming with the exact solving of the enigma of critical phenomena, and several hundred young physicists were entering the field with a great deal of excitement (Galam, 2004). The so-called modern theory of phase transitions along with 
renormalisation group techniques brought condensed matter physics into its golden age.

It is in this movement that Wilson developed his method of renormalisation, which provides insights into the field of critical phenomena and phase transitions and which enables exact resolutions. "The development of [the renormalisation group] technique undoubtedly represents the single most significant advance in the theory of critical phenomena and one of the most significant in theoretical physics generally" since the 1970s (Alastair \& Wallace, 1989, p. 237). We will briefly describe this method here in order to understand some of the connections econophysicists make with finance. Wilson's method considers each scale separately and then connects contiguous ones to one another. This makes it possible to establish a connection between the microscopic and the macroscopic levels by decreasing the number of interacting parts at the microscopic level until one obtains the macroscopic level (ideally a system with one part only). In this perspective, a complex system can be divided into $n$ levels in which the higher level is $n$.). Based on the statistical perspectives according to which the sum of the stable Levy distribution is still a stable Levy distribution, the renormalisation group method consists in using a scaling transformation to group the $k n$ random variables into $n$ blocks of $k$ random variables. The transformation $S_{n}$ takes the sequence $X$ into a new sequence of random variables, which is still independent and identically distributed. The transformation is called renormalisation group transformation with the critical exponent $\alpha$ while $j^{\text {th }}$ is the level of analysis. This transformation becomes truly fruitful when it is iterated, when each renormalisation leads to a reduction in the number of variables to give a system that contains fewer variables while keeping the characteristics of the original system - here the fact that the system stays independent, identically distributed and stable ${ }^{3}$.

Let us take an example of a system composed by 8 elements whose behaviour can be associated with variables $\left(\mathrm{X}_{1}, \ldots, \mathrm{X}_{8}\right)$. In this context, we have a sequence $X$ with $k n=8, n=4$ and $k=2$ which could be renormalized the sequence 3 times in order to obtain a single random variable that characterizes the initial sequence.

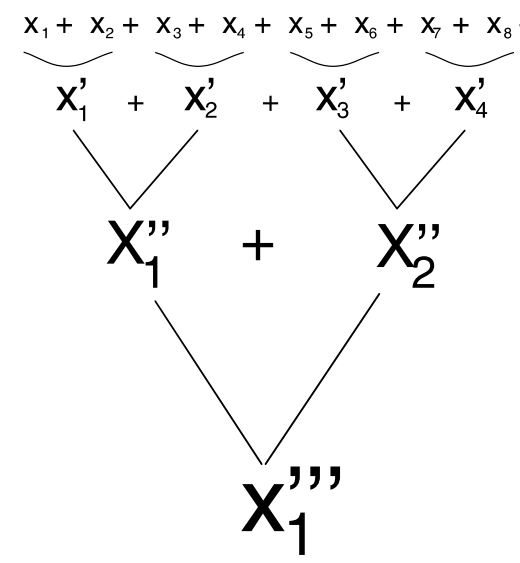

Figure 1: Renormalisation group method applied to a stochastic process (Sornette, 2006, $p$. 53)

Considering the renormalisation group method, the system at one scale consists of self-similar copies of itself when viewed at a smaller scale, with different parameters

\footnotetext{
${ }^{3}$ For more details, see Samorodnitsky and Taqqu (1994), Lesne (1998) or Sornette (2006).
} 
describing the components of the system. All scales are coupled to each other to generate the macro-level. In this context, physicists use a decoupling method taking the form of a scale invariance in order to study complex macro-phenomena (Cao \& Schweber, 1993). At the end of the 1970s statistical physics had established precise calculation methods for analysing phenomena characterised by scale invariance. Although the scale invariance assumption was not new in physics ${ }^{4}$, the properties allowing the mathematical demonstration of invariance were only established at this time. This demonstration makes it possible to study mathematically macroscopic regularities that occur and that are directly observable as a result of microscopic random interactions without to having study these microscopic interactions that are not directly observable. The focus is therefore on the direct phenomenological observation. In this perspective, scale invariance contributed to a better understanding of complex physical systems: physicists can calculate from knowledge of the microscopic constituents the parameters of generic models that allow the dynamic of macroscopic behaviours and then their evolution to be described without studying what happens at the microscopic level. For this reason, scale invariance is the contemporary foundation of any modern approach of statistical physics aimed at understanding the collective behaviour of systems with a large number of variables that interact with each other.

Research into critical phenomena and scale invariance have benefited from another very fruitful connection: the advent of the Ising model. The two-dimensional Ising model is a mathematical model of ferromagnetism used to study phase transition and critical points. This model is the simplest description of a dual system with a critical point. It played a central role in the development of research into critical phenomena and it occupies a place of importance in the mind of econophysicists. Precisely, at the critical point, the correlation length (i.e. the distance over which the direction of one spin affects the direction of its neighbour spins) is so important (and considered as infinite) that each spin is influenced by all neighbour spins. Due to the infinite correlation length, the spin system becomes scale invariant implying that the spin system has the same physical properties whatever the scale of the analysis. The renormalisation group method can then be applied, and by performing successive transformations of scales on the original system one can characterize the behaviour of the macro-system.

The Ising model has another very important feature: because of its very simple structure, it is not confined to the study of ferromagnetism. In fact, "[p]roposed as a model of ferromagnetism, it 'possesses no ferromagnetic properties' " (Hughes, 1999, p. 104)! Its abstract and general structure have enabled its use to be extended to the study many other problems or phenomena. For these reasons, statistical physicists consider the Ising model as the perfect illustration of a simple unifying mathematical model. Their looking for such models is rooted in the scientific view of physicists for whom "the assault on a problem of interest traditionally begins (and sometimes ends) with an attempt to identify and understand the simplest model exhibiting the same essential features as the physical problem in question" (Alastair \& Wallace, 1989, p. 237). The Ising model perfectly meets this requirement. Its use is not restricted to statistical physics because "the specification of the model has no specific physical content" (Hughes, 1999, p. 99) - this model is above all a

\footnotetext{
${ }^{4}$ For instance, it exists in the work of Euclid and Galileo.
} 
mathematical structure independent of the underlying phenomenon studied meaning it can be used to analyse any empirical data that share the same characteristics.

With these new theoretical developments (renormalization group theory and Ising model), statistical physicists have powerful mathematical models and methods for solving crucial problems in physics. Although the idea of establishing the behaviour of systems at their macroscopic level without analysing this microscopic level in details is not new in physics, physicists developed more and more specific tools improving this modelling of the macroscopic level. Moreover, the Ising model quickly appeared for physicists as a simple unifying mathematical model that can be used for the analysis of a large variety of problems. This progressive decontextualization of theoretical tools used in statistical physics contributed to their application outside of physics, as we will precise in the next section.

\subsection{The temptation to apply the methods of statistical physics outside physics}

Encouraged by the results obtained in the 1970s, certain physicists began investigating correspondences with collective behaviours of any kind of phenomena that appear critical, including social phenomena. For statistical physicists, as we will see now, this temptation to extend their models and methods outside physics seemed relevant because the methodological characteristic of the Ising model allow them to identify a universality class of problems. The first step was to identify a category of problems likely to be described through the same theoretical framework. Critical phenomena are a telling example of such category because they share the same behaviour at their critical points implying they can be grouped into the same universality class although they refer to very different realities - although these systems are microscopically different, they have identical macroscopic behaviour which is usually associated with the emergence of a statistical pattern. More specifically, the occurrence of critical phenomena is often statistically characterized through a power law in physics (Bak, 1987). In this perspective, the application of power laws for describing extreme phenomena in social sciences and finance makes sense for physicists since they have theoretical tools to deal with these phenomena.

This import of the renormalization group theory and the Ising model into social sciences is indicative of changes in the scientific methodology that occurred in the 20th Century. Giorgio Israel (1996) identifies a major change in the way of doing science through what he calls "mathematical analogies". These are based on the existence of unifying mathematical simple models that are not dedicated to the phenomena studied. Mathematical modelling is therefore used as mathematical analogies by means of which the same mathematical formalism is able to account for heterogeneous phenomena. The latter are "only interconnected by an analogy that is expressed in the form of a common mathematical description" (Israel, 1996, p. 41). In this perspective, the model is an effective reproduction of reality without ontology, one that may provide an explanation of phenomena. The Ising model is a perfect illustration of these simple unifying mathematical models. Israel (1996) stressed that such mathematical analogies strongly contribute to the increasing mathematisation of reality. 
Mathematical analogies support the temptations for statistical physicists to extend their models for analysing critical phenomena beyond physics. First, they looked for phenomena with large numbers of interacting units whose microscopic behaviours would not be observed directly but whose macroscopic results would be observed results that are consistent with the microscopic motions defined by a set of mathematical assumptions (which characterize random motion). Therefore, they looked for statistical regularities in order to identify scaling behaviours reflected by power laws and hence by a unifying mathematical model (Stanley \& Plerou, 2001, p. 563).

This approach led some statistical physicists to create new fields that were called "sociophysics" or "econophysics" depending the topics to which their methods and models were applied. A first movement, sociophysics, emerged in the 1980s (Galam, 2004; Săvoiu \& lorga-Simăn, 2013). Then in the 1990s statistical physicists turned their attention to economic phenomena, and particularly finance, giving rise to econophysics. Finance looked like the perfect candidate due to the theoretical hypotheses of the financial economics mainstream. Indeed, the cornerstone of financial economics, the efficient market theory, supposes that the financial markets components behave randomly and, according to Fama's reformulation in 1970, that investors can be represented by a representative agent. These two hypotheses are perfectly compatible with the statistical physics' approach that makes no hypothesis about specific behaviours of microscopic components except their random character ${ }^{5}$. Moreover, the renormalisation group method seems to be an appropriate answer to finance, because it provides a conceptual framework for understanding the macro-level of the systems (i.e. financial prices observed empirically) with minimal information from the micro-level (agents' behaviour). Thus, by analogy, statistical physicists view financial markets as complex systems, and prices as the statistical macro-result of a very large number of interactions at a theoretically defined microscopic level. This analogy has been supported by an important contextual element: the increasing use of computers in science and the computerisation of social reality that favoured the development of statistical physics outside of its original borders.

\subsection{The key role of computers}

Although independent of the theoretical developments that occurred in statistical physics, the computerization of social reality played a key role in the extension of statistical physics to social sciences for two reasons: 1) this computerization contributed to a better collection and exploitation of very large databases; 2) the main statistical tool (power laws) used by econophysicists require an enormous quantity of data to be identified due to their asymptotic properties

Computerisation has been implemented in a great number of fields dealing with social phenomena. Financial markets occupy a very specific place in this movement, because the financial databases are probably the largest sources of data for social phenomena. Indeed, since the end of the 1970s, all the major financial markets have been progressively automated thanks to computers. Automation has allowed all

\footnotetext{
5 The first agent-based models in econophysics did the "hypothesis [that] is similar to the 'representative agent' in Economics" (Chakrabortia et al., 2011, p. 1020).
} 
transactions and all prices quoted to be recorded. Then, since the 1990s, the evolution of technology paved the way to the development of high-frequency transactions, and therefore the creation of high-frequency data (also called "intraday" data). Previously, statistical data on financial markets were generally made up of a single value per day (the average price or the last quotation of the day). Nowadays, by recording "intraday data", computers keep in memory all prices quoted and tens of thousands of transactions traded every single day (R. Engle \& Russell, 2004).

The increasing quantities of data and the computerisation of financial markets led to notable changes. Intraday data brought to light new phenomena that could not be detected or did not exist with monthly or daily data. The most important for our subject is that these new intraday data have exhibited more extreme values than one could be detected before. Indeed, before the computerisation, prices recorded were estimated through a simple mean of prices used for transactions during the day. In this context, extreme values were smaller and less frequent than in intraday data. Consequently, intraday data have generated three major changes: 1) they have increased the interest for research on extreme values; 2) they have brought new challenges in the analysis of stock price variations that have required the creation of new statistical tools to characterise them; 3) they favour the growing interest for extending the methods and models of statistical physics, which are based on huge amounts of data, into finance. The advent of intraday data has made it possible to build samples that are sufficiently large to provide empirical evidence for supporting the application of power-law distribution analysis to the evolution of prices $\backslash$ returns. According to some authors (Gallegati, et al., 2006b, p. 1) this expansion of financial data - which has no equivalent in other social sciences fields - makes financial markets "a natural area for physicists".

Computers have also transformed scientific research on distributions of stock-market variations in a very fruitful way for power-laws. Their ability to perform calculations more rapidly than human beings opened the door for analysis of new phenomena and this situation also allows old phenomena to be studied in a new ways. This is particularly true for stable Lévy processes. In general, there are no closed-form formulas for stable Lévy distributions - except in their Gaussian, Pareto and Cauchy forms - which makes them difficult to work with. Working with such distributions implies a specific parameterization requiring complex calculations with numerous data. Computer simulations have changed the situation because they allow research when no analytic solution exists by making possible to chart step-by-step the evolution of a system whose dynamics are governed by non-integrable differential equations (i.e. which no analytic solution). Computerisation has also provided a more precise visual analysis of empirical data: indeed, by allowing simulations for different parameters of the stable Lévy distributions, computers have paved the way to a visual research that could have appeared vague before (Mardia \& Jupp, 2000). It is worth mentioning that computers have also facilitated work with stable Lévy distribution since several statistical and mathematical programs have been developed to compute stable densities, cumulative distribution functions and quantiles, resolving most of computational difficulties usually associated with stable Lévy distributions ${ }^{6}$.

\footnotetext{
${ }^{6}$ We can mention ModEco (http://modeco-software.webs.com/econophysics.htm) developed by a retired academic physicist or Rmetrics (https://www.rmetrics.org/) developed by Econophysics Group from the University of Zurich - EHT Zurich. It is worth mentioning that the latter software is directly
} 


\section{A new tool for analysing extreme values: power-law distributions}

After having clarified the elements that contributed to the extension of physics outside of its borders, we will present in this section the conceptual framework used by econophysics, and more specifically, the theoretical foundations of power laws in statistical physics. Afterwards, we will discuss their extension in finance. The majority of works related to econophysics claim that the financial markets (or economic systems) can be considered as complex system whose evolution of the macrobehaviour follows a power-law distribution (also often referred to as heavy-tail distributions, Pareto distributions, Zipf distributions, etc. $)^{7}$. A finite sequence $y=\left(y_{1}\right.$, $\left.y_{2}, \ldots, y_{n}\right)$ of real numbers, assumed without loss of generality always to be ordered such that $y_{1} \geq y_{2} \geq \ldots \geq y_{n}$, is said to follow a power law if

$$
k=c y_{k}^{-\alpha} \text {, }
$$

where $\mathrm{k}$ is (by definition) the rank of $y_{k}, c$ is a fixed constant, and $\alpha$ is called the critical exponent or the scaling parameter. In case of a power-law distribution, the tails decay asymptotically according to $\alpha$ - the smaller the value of $\alpha$, the slower the decay and the heavier the tails. A more common use of power laws occurs in the context of random variables and their distributions. That is, assuming an underlying probability model $P$ for a nonnegative random variable $X$, let $F(x)=P[X \leq x]$ for $x \geq 0$ denote the (cumulative) distribution function of $X$, and let $\bar{F}(x)=1-F(x)$ denote the complementary cumulative distribution function. In this stochastic context, a random variable $X$ or its corresponding distribution function $F$ is said to follow a power law or is scaling with index $\alpha>0$ if, as $x \rightarrow \infty$,

$$
P[X>x]=1-F(x) \approx c x^{-\alpha},
$$

for some constant $0<c<\infty$ and a tail index $\alpha>0$. For $1<\alpha<2, F$ has infinite variance but finite mean, and for $0<\alpha \leq 1, F$ has infinite variance and infinite mean. In general, all moments of $F$ of order $\beta \geq \alpha$ are infinite.

The importance of power laws in statistical physics did not pop up from nowhere. The previous section explained their links with critical phenomena, which have largely monopolized the interest of physicists in the last decades. This interest has created a kind of fascination of power laws for econophysicists:

"Why do physicists care about power laws so much? [...] The reason [...] is that we're conditioned to think they're a sign of something interesting and complicated happening. The first step is to convince ourselves that in boring

used in university modules developed by this group (https://www.rmetrics.org/sites/default/files/2013VorlesungSyllabus.pdf). More famous statistical and mathematical software gradually integrate key econophysics concepts. The well-known Mathemactica, for example, has proposed a "stable distributions package" for 2005 (Rimmer \& Nolan, 2005). Aoyama et al. (2011) showed that the statistical software Stata and SAS can also be used for an econophysical analysis of economic data. We can also mention Alstott et al. (2014), or the script provided by Aaron Clauset in his web page, http://tuvalu.santafe.edu/ aaronc/powerlaws/, to be used with Matlab.

${ }^{7}$ There are some exceptions, particularly in the most recent works (Nakao, 2000). 
situations, we don't see power laws" (Shalizi's notebook http://bactra.org/notebooks/power-laws.html).

Bouchaud (2001, p. 105) expresses a similar idea:

"Physicists are often fascinated by power laws. The reason for this is that complex, collective phenomena give rise to power laws which are universal, that is, to a large degree independent of the microscopic details of the phenomenon. These power laws emerge from collective action and transcend individual specificities. As such, they are unforgeable signatures of a collective mechanism".

Such fascination has strongly contributed to the increasing number of empirical studies on power laws.

\subsection{Power-law distributions seen as a phenomenological law}

First at all, we must mention that empirical investigations into phenomena distributed according to power laws are nothing new in economics. They date back to Pareto (1897) and, since then, they have been regularly observed and studied for numerous phenomena since (Mitzenmacher, 2004; Simkin \& Roychowdhury, 2011). Statistical physicists thus joined a larger movement, reinforcing the idea that power laws and critical phenomena constitute important tools for analysing empirical phenomena.

The common way to probe for power-law behaviour has consisted in checking visually on a simple histogram that the frequency distribution of the quantity of $x$ appears as a straight line when plotted on double logarithmic axes. Indeed, taking the logarithm of both sides of the equation 1, we see that the power-law distribution obeys In $P[r>x]=-\alpha \ln x+c$. Thus, a distribution that approximately falls on a straight line provides an indication that the distribution may follows a power law, with a scaling parameter $\alpha$ given by the absolute slope of the straight line. This type of visual investigation has guided econophysicists' empirical research. This linearity has been observed in a wide variety of phenomena, suggesting that power law distribution could fit with a great number of observations. Moreover, the number of observations has considerably increased with the spread of computerized databases (Dubkov et al., 2008). Thus, "[i]n the mid-1990s, when large data sets on social, biological and technological systems were first being put together and analyzed, power-law distributions seemed to be everywhere [...]. There were dozens, possibly hundreds of quantities, that all seemed to follow the same pattern: a power-law distribution"

(http://tuvalu.santafe.edu/ aaronc/courses/7000/csci7000-

0012011 L3.pdf). The result was that some "scientists are calling them more normal than normal [law] [; and therefore] the presence of power-law distributions in data [...] should be considered as the norm rather than the exception" (Willinger cited in Mitchell, 2009, p. 269). This linear relationship was also observed in financial and economic phenomena in addition to Pareto's observations on income distribution (Axtell, 2001; Cordoba, 2008; Eeckhout, 2004; Gabaix, 2009; Gabaix et al., 2007; Gabaix \& Landier, 2008; Klass et al., 2006; Krugman, 1996; Levy, 2003; Lux, 1996). Mandelbrot was the first to identify it in stock-price variations, and he applied the stable Lévy process to stock-price movements in the early 1960s. Although financial economists did not follow Mandelbrot's research (after some failed 
attempts in the 1960s and the 1970s due to mathematical difficulties ${ }^{8}$ ), economists have always used power laws as a descriptive framework to characterise some economic phenomena - such as the relationship between the size of firms, cities or organizations with one of their characteristics (Jovanovic \& Schinckus, 2016). Recently, Gabaix (2009) showed that the returns of the largest companies on the New York Stock Exchange exhibit the same visual linearity.

The high number of empirical data suggests a kind of the phenomenological universality for power law. In addition, these observations have supported the idea that methods and models coming from statistical physics could be applied outside physics. However, it is worth mentioning that the association of these empirical regularities with a power law is not free of theoretical presuppositions that are directly inherited from physics as explained hereafter.

\subsection{The theoretical foundations of power laws in statistical physics}

The major theoretical foundation given to the observations of power refers to the self-criticality theory introduced, in physics, by Peter Bak et al. $(1987,1988)$. Although this theory has originally been developed to describe the emergence of power laws characterizing the evolution of physical systems, it is often used by econophysicists to defend the existence of such macro-laws in the finance area. According to Bak, the linearity visually identified in the histogram plotted on a log-log axes, can be interpreted as the expression of the complexity of a phenomenon (Bak, 1994, p. 478). The basic idea of self-organized criticality is that certain phenomena maintain themselves near a critical state. Physicists talk about "critical state" because the system organizes itself into a fragile configuration based on a knifeedge $^{9}$. Although their instability, some complex systems look to be ruled by a single macroscopic power-law describing the frequency at which phase transitions occur (Newman, 2005). This theory of self-organized criticality progressively became a theoretical reference for physicists for who power laws are synonymous with complex systems. The importance of power laws in the analysis of complex systems can be understood at three levels: 1) the distribution describing the occurrence of critical states and correlation lengths; 2) these laws have scaling properties and; 3) these laws refers to a large variety of phenomena invariance (universality classes).

First level, the correlation lengths: for critical phenomena, the large correlation lengths that exist in the system at the critical point are distributed like a power law. Traditionally, physicists characterised the correlations between the constituents of the system with an exponential law $\mathrm{e}^{\frac{-\mathrm{r}}{\xi(\mathrm{T})}}$ (i.e. the correlation function), where $r$ is the distance between two points and $\xi(\mathrm{T})$ is the correlation length - precisely, $\xi(\mathrm{T}) \propto$ $|\mathrm{T}-\mathrm{Tc}|^{-v}$. Then, following the observations of critical phenomena, in order to characterise the divergence observed at the critical point, they added to the

\footnotetext{
${ }^{8}$ See Jovanovic and Schinckus (2013) for further details on the topic.

9 The basic idea of self-organized criticality is that certain phenomena maintain themselves near a critical state. A telling example of that situation is a quiet sand pile in which the addition of one grain generates mini-avalanches. At some point, these mini-cascades stop meaning that the sand pile has integrated the effect of this additional gran. The sand pile is said to reach its self-organized critical state (because the addition of a new sand grain would generate the same process). Physicists talk about "critical state" because the system organizes itself into a fragile configuration based on a knifeedge (the addition of only one sand grain would be enough to modify the sand pile).
} 
exponential law a power law: $\mathrm{r}^{-\alpha} \mathrm{e}^{\frac{-\mathrm{r}}{\overline{\xi(T)}}}$. Therefore, at the critical point, due to the divergence, the exponential, $\mathrm{e}^{\frac{-\mathrm{r}}{\xi(\mathrm{T})}}$, goes to zero and the correlation function is distributed according to a power law alone, $\mathrm{r}^{-\alpha}$. In other words, away from the critical point the correlation length between two constituents, $x$ and $y$, decays exponentially, $\mathrm{e}^{\frac{-|x-y|}{\xi(T)}}$. But when we approach the critical point, the correlation length increases, and right at the critical point, the correlation length goes to infinity and decays in accordance with the power of the distance, $|x-y|^{-a}$. Intuitively, far from the critical point, the microscopic elements dynamics approximately follow a Gaussian distribution but, when we approach the critical point, this microscopic dynamics can deliver a non-Gaussian stationary distribution.

Second level, the scaling properties: at their critical point, the phenomena become independent of the scale used; showing therefore a scaling invariance. The lack of a characteristic scale implies that the microscopic details do not have to be considered in the analysis. Scaling invariance is the footprint of critical points - and of critical phenomena too - and power-law distribution is the sole distribution that has a scale invariance property. In other words, at the critical point, "the observable quantities in the system should adopt a power-law distribution" (Newman, 2005). This means that the shape of the phenomenon's size distribution curve does not depend on the scale on which we measure the size of the phenomenon (the results are exactly the same for "small" scale as for "large" scale). For this reason, power laws are called scaling laws.

Third level, the universality classes. This connection comes from the critical exponent that characterises power laws. Determining the critical exponent of a phenomenon allows this phenomenon to be associated with a specific universality class. This association serves to identify some characteristics of the system used to model the phenomenon and to deduce their behaviour at their critical point. Moreover, the classification of critical phenomena into universality classes is crucial in finance, because stability is only guaranteed when we work within a single universality class (adding two stable random variables with different critical exponents does not give a stable distribution).

These three elements have provided theoretical foundations for the use of power laws outside of physics. When physicists observe a power law characterizing the dynamics of no-physical systems, they implicitly import the theoretical tools they usually associated with these statistical regularities. So doing, physicists propose a metaphorical analogy in line with what we explained in the section 3.2.

\subsection{Power laws and the framework of financial economics}

Power laws have at least three crucial connections with the theoretical framework of financial economics.

First connection, power laws are easily deduced from the financial definition of returns. Considering the price of a given stock, $p_{t}$, the stock return $r_{t}$ is the change of the logarithm of the stock price in a given time interval $\Delta t$,

$$
r_{t}=\ln p_{t}-\ln p_{t-\Delta t}
$$


Therefore, the probability of having a return $r$ higher than the return $x, P[r>x]$, can be written as $\ln P[r>x]=-\alpha \ln x+c$, which can be rewritten as a power law expression by using the exponential of both sides of the equation, $P[r>x]=c x^{-\alpha}$.

Second connection, power laws are easily linked with the stochastic processes used in financial economics, which describe the evolution of a variable $X$ (price, return, volume etc.) over time $(t)$. Knowing that a power law is a specific relationship between two variables that requires no particular statistical assumptions about these two variables, the evolution between the variables $X$ and $t$ may be associated with a power law. In this case, this evolution is a stable Levy process such as

$$
P(x) \sim \frac{C}{|x|^{1+}}(\text { for } x \rightarrow \pm \infty)
$$

where $C$ is a positive constant called the tail or scale parameter and the exponent $\mu$ is between 0 and $2(0<\mu \leq 2)$. It is worth mentioning that among Lévy processes, only stable Lévy processes can be associated with power laws because the stability property is a statistical interpretation of the scaling property. While stochastic processes used in financial economics are mainly based on the Gaussian framework, taking a Gaussian process as a starting point, a no-normal diffusion law can be obtained by keeping the independence and the stationarity of increments but by characterising their distribution through a large law (i.e. exhibiting fat tails).

Third and last connection, the interest of econophysics for finance is related to the work of Mandelbrot. This author attempted to extend statistical physics to other fields, including social sciences since the 1960s (Mandelbrot, 1957, p. 4). He provided crucial results for financial economics, particularly by suggesting, with Samuelson (1965), to replace the random-walk model with the martingale model (Mandelbrot, 1966a). Martingale model is a cornerstone for the work of Harrison and Kreps (1979), Kreps (1981) and Harrison and Pliska (1981) that gives a mathematical definition of the theory of informational efficiency. Thus, in a sense, econophysicists, who refer systematically to Mandelbrot's work, seem to have concretized his project - we will discuss this link in the next section.

\section{Breaking down the disciplinary barrier between econophysics and financial economics}

The previous sections have explained the relevance of power laws in finance; they also showed that some conceptual and historical links between econophysics and financial economics exist. In this context, the question is: why these two fields do not propose a more integrative collaboration. Why are econophysics works mainly published in physics? We can identify four barriers explaining the difficulty for econophysicists to be published in financial journals: 1) the different expectations in the publishing process between econophysicists and financial economists; 2) the confusion due to the use of concepts and vocabulary in different ways; 3 ) the lack of generative models (financial meaning) explaining the emergence of power laws; and 4 ) the lack of quantitative tests for identifying power laws. 


\subsection{Different scientific cultures}

The first reason of this no-collaboration is simply related to the different scientific culture between the two communities (Jovanovic \& Schinckus, 2016, p. chap. 4; McGoun, 2003, p. 432). Papers dedicated to econophysics are mainly published in physics journals implying that their vocabulary, method, and models are those used in physics (Gingras and Schinckus, 2012). Although scientific papers appear contextless, they are social constructions referring to a disciplinary culture based on an implicit knowledge shared by scientists involved in that discipline. In their scientific structure, articles published in a specific field share a highly stylised and a formal system of presentation that aim to convince readers who expect to find this specific system (Bazerman, 1988; Gilbert \& Mulkay, 1984; Knorr-Cetina, 1981, p. chap. 5). For instance, financial economists and physicists tend to present their scientific writings differently. A common practice in financial economics is to write a substantial literature review "demonstrating the incrementalism of this literature" (Bazerman, 1988, p. 274) in order to emphasise the accumulation of knowledge and the ability of authors to contribute to a pre-existing codified knowledge. In contrast, physicists mainly focus on the practical implications of their articles, mentioning only references that deal with potential applications (Jovanovic \& Schinckus, 2016). Consequently, due to these differences, it is more difficult for econophysicists to get past the gatekeepers of financial economics journals. However, beyond this observation, we must stress that in recent years more and more joint publications (such as this special issue, for example) and research have been developed, taking part in bridging the gap between these two scientific cultures (Ausloos, 2001; Bouchaud, 2002; Carbone et al., 2007; Chakrabarti \& Chakraborti, 2010; Chen \& Li, 2012; Durlauf, 2005; Farmer \& Lux, 2008; Gabaix, 2009; Keen, 2003; Lux, 2009; McCauley, 2006; Stanley \& Plerou, 2001).

\subsection{The lack of common referent}

As Jovanovic and Schinckus (2016) explain, econophysics and financial economics use similar concepts but in different ways, creating confusions and misunderstandings. We can illustrate that point with the connexion between Mandelbrot and econophysicists mentioned in the previous section. While both arrive at the same result - modelling stock-price variations using stable Lévy processes, they do not take the same path to get there. Mandelbrot starts his analysis from a purely mathematical property: the stability of stochastic process, which is, according to this author, the most important hypothesis for a process in order to produce new interesting results in finance (Mandelbrot, 1966b). In this context, his suggestion was to generalise Gaussian processes by using stable Lévy distributions and the generalised central-limit theorem, which is compatible with stable Lévy distribution.

The origin of power law used by econophysicists is quite different since their interest in this statistical framework rather results from the characterization of critical phenomena through the renormalisation group method. (Lesne \& Laguës, 2012, p. 63). Renormalisation method allows the demonstration of the stability for nonGaussian stable processes. More precisely, the renormalisation method is based on the property of being distributed according to a power law is conserved under 
addition, multiplication, and polynomial transformation ${ }^{10}$. Moreover, the renormalisation group methods focus on the scaling property of the process, which a characteristic of critical phenomena.

This conceptual difference between Mandelbrot's works and contemporary studies in econophysics explains why authors involved in the latter work with power law distributions while Mandelbrot only focused on stable Lévy distributions. Actually, the latter is a specific case of the first since stable Lévy distribution is associated with a power law whose increments are independent. In other words, econophysicists and Mandelbrot use the same statistical tools but not for the same theoretical reasons. Furthermore, this observation reminds that econophysics is an original research and contradicts the argument of the no-originality of econophysics usually claimed by economists who presented this field as a simple extension of of Mandelbrot's works.

\subsection{The lack of quantitative tests}

The third important barrier refers to the absence of real statistical tests for the identification of power laws. The vast majority of existing statistical tools have been developed in a Gaussian framework making them inappropriate to test power laws. Scientists know that this problematic issue makes difficult the empirical use of power laws (Mitzenmacher, 2005; Newman, 2005). To date, and as we explained before, econophysicists based their empirical results on a visual technique for identifying that a phenomenon fits with a power law. This visual approach is extremely problematic for financial economists for two major reasons. Firstly, this method generates significant systematic errors (Clauset et al., 2009; Gillespie, 2014; Stumpf \& Porter, 2012), particularly because power laws can visually be close to so-called exponential laws. Only a large volume of data makes it possible to distinguish between the two types of law (Mitzenmacher, 2004). Moreover, the visual approach has no objective criterion for determining what a "good fitting" is. For instance, LeBaron (2001) also showed that simple stochastic volatility models can produce similar behaviours to those obtained by econophysicists with power laws. Stanley and Plerou (2001) replied to LeBaron's critique, but, although their reply provided an interesting technical reply, it also showed the methodological difficulties between the two approach. Secondly, financial economists, who are defenders of quantitative tests, created their own discipline by rejecting strongly the visual approach used for predicting stock price variations (Jovanovic, 2008). They promoted the use of quantitative tests as scientific condition for their emerging discipline. Considering this historicallmethodological position, visual tests used by econophysicists are considered by financial economists as having no credibility, and even no scientific foundations.

The lack of quantitative tests makes econophysics literature hardly acceptable to financial economists (Durlauf, 2005, p. F234). Moreover, the problems created by the visual approach are also well known by other scientific communities (Mitzenmacher, 2004) but also by some econophysicists (Clauset, et al., 2009, p. 691), who pointed out that "better and more careful testing is needed, and that too

\footnotetext{
${ }^{10}$ When we combine two power-law variables, the one with the fatter-tailed distribution (that is, the one with the smaller exponent) dominates. The new distribution is the minimum of the tail exponents of the two distributions combined.
} 
much of data analysis in this area relies on visual inspection alone" (Farmer \& Geanakoplos, 2008, p. 24).

Providing a quantitative method for testing power law is a big challenge from a mathematical and statistical viewpoint, and very few authors have been working on. Moreover, from a financial economics' viewpoint, several problems exist in order to develop statistical tests dedicated to power laws (Broda et al., 2013, p. 293). Despite these difficulties, in the recent years, significant results have emerged. The literature has taken two different approaches to test power laws: on the one hand, some authors have used a rank-size rule (Gabaix \& Ibragimov, 2011); and, on the other hand, some authors have worked on the size-frequency relation (Clauset, et al., 2009). However, to date these tests are not applied to financial time series. Nevertheless, due to the rapid expansion of statistical studies on this topic, we could expect the development of a new research in the close future ${ }^{11}$.

\subsection{Two ways of defining the explanation}

Although econophysicists have obtained numerous empirical observations during several decades, there are no generative models explaining the emergence of power laws in economic terms. From the statistical physics' viewpoint, we could consider that seeing phenomenon studied as a critical phenomenon is a theoretical justification for the use of models coming from statistical physics. However, leaving this theoretical argument aside, econophysicists have produced no generative models to explain why power laws govern the economic phenomena studied. This situation also existed in the other fields in which statistical physicists have extended their models and methods (Mitzenmacher, 2005). The observation of a statistical law per se does not give a meaning to the observed data and although econophysicists refers to what makes sense for them, they do not provide an "understandable meaning" which could make sense for an economists as Durlauf (2005, p. F235) pointed this out. Consequently, "The econophysics approach to economic theory has generally failed to produce models that are economically insightful" (Durlauf, 2005, p. F236). This problem is reinforced by the fact that econophysicists and economists have a different meaning for what is an acceptable explanation ${ }^{12}$ : the first use the renormalization group theory and scaling invariance as theoretical foundations for their macro-explanation of financial markets while economists rather explain these markets through a microscopic incentives-based approach ${ }^{13}$. Econophysicists propose a statistical explanation (based on a decoupling scales method) while financial economists use a behaviourist explanation founded on an assumption of agents' perfect rationality. In other terms, the two communities are working in two very different conceptual worlds. This situation helps to explain the reason for why financial economists perceive as "insightful" the works developed by econophysics considering that these works do not have a theoretical framework behind their

\footnotetext{
${ }^{11}$ See Jovanovic and Schinckus (2016) for further details on the alternative issues which can be investigated in order to provide quantitative tests for the power laws.

12 This kind of difference echoes to the historical debates between the Newtonian physics and the Cartesian Physics: while the first "explained" everything in terms of gravitational force, the latter rather "explained" phenomena through a mechanical framework. See Gingras (2001) for further details about this debate.

${ }_{13}$ The microscopic (individual) perspective is very important in economics and finance even for all issues related to macroeconomics. It is worth reminding that since Lucas' critic (1976), it has been widespread accepted that macroeconomics must be based on microeconomic principles.
} 
statistical sophistication. However, the previous sections of this article showed that the problem is not the lack of theoretical explanations but rather the misunderstood between the theoretical world within econophysicists and financial economists work.

Actually, the lack of generative models constitutes a serious barrier between the two fields because the financial economists largely - we could even say almost exclusively - base their works on models with theoretical explanation. Simulations of real phenomena without theory are considered as very weak results for economists who already had methodological debates about the irrelevance of a strictly datadriven approach in their field - one can mention the Koopmans-Vining debate at the end the 1940s (Mirowski, 1989a), or more recently, the Real Business Cycle (RBC) models approach (Eichenbaum, 1996; Hansen \& Heckman, 1996; Hoover, 1995; Quah, 1995; Sims, 1996). The necessity for a theoretical explanation could lead to paradoxical situations: on one hand, it contributes to keep the efficient market theory despite its weak connection with the random character of stock price variations (Jovanovic, 2010); on the other hand, it contributes to justify the ARCH class of models despite it is a purely statistical approach providing no explanation of the empirical regularities" (Lux, 2006). In this context, ARCH models appear to be very similar to the way of modeling in econophysics implying that the reject of econophysics' works is not plenty comprehensible from the econophysicists' viewpoint. However, from a financial economics viewpoint two nuances exist although these nuances could seem very tenuous - Firstly, ARCH class of models uses statistical tests. Secondly, these models were used in finance in the perspective to test the efficient market theory while this theory is hardly testable (Jovanovic, 2010). Therefore, most of financial economists using ARCH class of models consider their models have theoretical foundations from a financial (and not from statistical only) point of view.

In this perspective, a generative model shedding the light on the potential reasons for why power laws emerge in finance would represent a major step in the perspective of an integrated approach between financial economics and econophysics. Although models explaining the emerge of power laws in finance are not numerous (the vast majority of empirical works show the existence of a power law without explaining its origin), one can mention the works of Gabaix et al. (2006; 2003) showing how institutional investor's trades have an impact on the evolution of financial prices and volatility and generate a fat-tailed distribution of volumes and returns. The starting point of their model is the observation of the distribution related to the investors' size, which would takes the form of a power law ${ }^{14}$. That fat-tailed distribution means that we can observe a big difference between large and small trading institutions implying an important heterogeneity of actors (in terms of size). From this diversity, it results a dispersal of the trading power in which only bigger institutions will have a real impact on the market. They demonstrated how an optimal trading behavior of large institutions (considering that these latter are the only ones that have an impact on the market prices) can generate a power law in the distribution of trading volume and financial returns. Gabaix et al. $(2003,2006)$ model constitutes a crucial step in very recent contributions concerning generative models

\footnotetext{
${ }^{14}$ We can notice that in contrast Farmer et al. (2004) have shown that large price changes in response to large orders are very rare. See also Chiarella et al. (2009) for a more recent model showing that large price changes are likely to be generated by the presence of large gaps in the book of orders.
} 
in order to explain the power law behaviors in financial data. We can also mention some works developed in the literature dealing with agent-based modelling which tried to identify an origin to the power-laws in finance: Lux and Marchiesi (2000), Alfarano et al. (2008) or Feng et al. (2012) proposed a specific calibration of microinteractions for models whose iterations can reproduce the observed power laws. We can wish this work paved the way for the development of potential explanative models for the emergence of power laws in financial data.

\section{Conclusion: What remains to be done...}

This article has studied the main reasons for why economists have difficulties to recognize the works developed by econophysicists. Beyond the clarification of the current situation between these two communities, this paper also pointed out many crucial progresses that took place in the recent years. Moreover, it leads to identify what remains to be done (from an economist point of view) for econophysicists to contribute significantly to financial economics and for a future collaboration between econophysics and financial economics. As we saw, three paths have still waiting to be investigated.

A proposed agenda would be to develop 1) a common frameworklvocabulary in order to better compare and integrate the two approaches; 2) statistical tests in order to identify and to test the power laws or, at least, to provide statistical tests to compare results from econophysics' models with those given by financial models; 3 ) generative models in order to give a theoretical explanation for the emergence of power laws.

The major objective of this paper was to clarify the reasons of the current dialogue of deaf between economists and econophysicists. This study led us to present the contextual elements, which contributed to the extension of physics outside of its borders. Afterwards, we presented the theoretical foundations supporting this extension in economics. This clarification allowed us to suggest a research agenda for a future fruitful collaboration between econophysicists and financial economists. Of course, this suggested agenda will certainly raise a number of questionslchallenges but it creates many research opportunities by improving collaborations between financial economists and econophysicists.

\section{References}

Alastair, B., \& Wallace, D. 1989. Critical phenomena: universal physics at large length scales. in P. Davies (Ed.), The new Physics, Cambridge University Press, Cambridge.

Alfarano, S., Lux, T., \& Wagner, F. 2008. Time variation of higher moments in a financial market with heterogeneous agents: an analytical approach. Journal of economic dynamics \& control 32, 101-136.

Alstott, J., Bullmore, E., \& Plenz, D. 2014. Powerlaw: a Python package for analysis of heavy-tailed distributions. PloS one 9, e85777.

Aoyama, H., Fujiwara, Y., Ikeda, Y., Iyetomi, H., \& Souma, W. 2011. Econophysics and Companies: Statistical Life and Death in Complex Business Networks. Cambridge University Press, Cambridge. 
Ausloos, M. 2001. Special issue on Econophysics. The European Physical Journal B 20, 471-471.

Axtell, R. L. 2001. Zipf distribution of U.S firm sizes. Science in Context 293, 18181820.

Bak, P. 1994. Introduction to self-criticality. in G. Cowan, D. Pines \& D. Meltzer (Eds.), Complexity: Metaphors, Models, and Reality, Vol. 19, Santa Fe Institute, Santa Fe, pp. 476-482.

Bak, P., Tang, C., \& Wiesenfeld, K. 1987. Self-organized criticality: an explanation of 1/f noise. Physical Review Letters 59, 381-384.

Bak, P., Tang, C., \& Wiesenfeld, K. 1988. Self-Organized Criticality. Physical Review A 38, 364-374.

Ball, P. 2006. Econophysics Culture Crash. Nature 441, 686-688.

Bazerman, C. 1988. Shaping Written Knowledge: The Genre and Activity of the Experimental Articles in Science. University of Wisconsin Press, Madison.

Bouchaud, J.-P. 2001. Power laws in economics and finance: some ideas from physics. Quantitative Finance 1, 105-112.

Bouchaud, J.-P. 2002. An Introduction to statistical finance. Physica A 313, 238-251.

Bouchaud, J.-P., Mezard, M., \& Potters, M. 2002. Statistical properties of stock order books: Empirical results and models. Quantitative Finance 2, 251-256.

Broda, S. A., Haas, M., Krause, J., Paolella, M. S., \& Steude, S. C. 2013. Stable mixture GARCH models. Journal of Econometrics 172, 292-306.

Cao, T. Y., \& Schweber, S. S. 1993. The Conceptual Foundations and the Philosophical Aspects of Renormalization Theory. Synthese 97, 33-108.

Carbone, A., Kaniadakis, G., \& Scarfone, A. M. 2007. Where do we stand on econophysics. Physica A 382, 11-14.

Chakrabarti, B. K., \& Chakraborti, A. 2010. Fifteen Years of Econophysics Research.

Chakrabortia, A., Muni Tokea, I., Patriarcabc, M., \& Abergela, F. 2011. Econophysics review: I. Agent-based Models. Quantitative Finance 11, 1013-1041.

Chen, S.-h., \& Li, S.-p. 2012. Econophysics: bridges over a turbulent current. International review of financial analysis 23, 1-10.

Chiarella, C., Iori, G., \& Perello, J. 2009. The impact of heterogeneous trading rules on the limit order book and order flows. Journal of Economic Dynamics \& Control 33, 525-537.

Clauset, A., Shalizi, C. R., \& Newman, M. 2009. Power-law distributions in empirical data. SIAM Review 51, 661-703.

Cont, R., \& Tankov, P. 2004. Non-parametric calibration of jump-diffusion option pricing models. Journal of Computational Finance 7, 1-49.

Cordoba, J. 2008. On the distribution of city sizes. Journal of Urban Economics 63, 177-197.

Daniel, G., \& Sornette, D. 2010. Econophysics: historical perspectives. in C. Rama (Ed.), Encyclopedia of Quantitative Finance, Wiley, London.

Davis, M., \& Etheridge, A. 2006. Louis Bachelier's Theory of speculation. Princeton university press, Princeton and Oxford.

Donangelo, R., \& Sneppen, K. 2000. Self-organization of value and demand. Physica A 276, 572-580.

Dubkov, A. A., Spagnolo, B., \& Uchaikin, V. V. 2008. Lévy Flight superdiffusion : an introduction. International Journal of Bifurcation and Chaos 18, 2649-2672.

Durlauf, S. N. 2005. Complexity and Empirical Economics. Economic Journal 115, F225-F243. 
Eeckhout, J. 2004. Gibrat's law for (all) cities. American Economic Review 94, 1429_ 1451.

Eichenbaum, M. 1996. Some comments on the role of econometrics in economic theory. Economic Perspectives 20, 22.

Engle, R., \& Russell, J. 2004. Analysis of high frequency financial data. in University of Chicago Working Paper.

Engle, R. F. 1982. Autoregressive Conditional Heteroscedasticity with Estimates of Variance of United Kingdom Inflation. Econometrica 50, 987-1008.

Farmer, J. D., \& Geanakoplos, J. 2008. Power laws in economics and elsewhere. in Working paper.

Farmer, J. D., Gillemot, L., Lillo, F., Mike, S., \& Sen, A. 2004. What really causes large price changes? Quantitative Finance 4, 383-397.

Farmer, J. D., \& Lux, T. 2008. Introduction to special issue on 'Applications of Statistical Physics in Economics and Finance'. Journal of Economic Dynamics and Control 32, 1-6.

Feng, L., Li, B., Podobnik, B., Preis, T., \& Stanley, H. E. 2012. Linking agent-based models and stochastic models of financial markets. Proceedings of the National Academy of Sciences of the United States of America 109, 83888393.

Fitzpatrick, R. 2012. Thermodynamics \& Statistical Mechanics: An intermediate level course. in.

Francq, C., \& Zakoian, J.-M. 2010. GARCH Models: Structure, Statistical Inference and Financial Applications. Wiley, London.

Gabaix, X. 2009. Power Laws in Economics and Finance. Annual Review of Economics 1, 255-293.

Gabaix, X., Gopikrishnan, P., Plerou, V., \& Stanley, H. E. 2006. Institutional investors and stock market volatility. Quarterly Journal of Economics 121, 461-504.

Gabaix, X., Gopikrishnan, P., Plerou, V., \& Stanley, H. E. 2007. A unified econophysics explanantion for the power-law exponents of stock market activity. Physica A 382, 81-88.

Gabaix, X., Gopikrisnan, P., Plerou, V., \& Stanley, H. E. 2003. A theory of power law distributions in financial market fluctuations. Nature 423, 267-270.

Gabaix, X., \& Ibragimov, R. 2011. Rank - 1/2: A Simple Way to Improve the OLS Estimation of Tail Exponents. Journal of Business \& Economic Statistics 29, 24-39.

Gabaix, X., \& Landier, A. 2008. Why has CEO pay increased so much? Quarterly Journal of Economics 123, 49-100.

Galam, S. 2004. Sociophysics: a personal testimony. Physica A 336, 49-55.

Gallegati, M., Keen, S., Lux, T., \& Ormerod, P. 2006a. Worrying trends in econophysics. Physica A: Statistical Mechanics and its Applications 370, 1-6.

Gallegati, M., Keen, S., Lux, T., \& Ormerod, P. 2006b. Worrying trends in econophysics. Physica A 370, 1-6.

Gilbert, G. N., \& Mulkay, M. 1984. Opening Pandora's box. Cambridge University Press, London and New York.

Gillespie, C. S. 2014. A complete data framework for fitting power law distributions. in.

Gingras, Y. 2001. What Did Mathematics Do to Physics? History of Science 39, 383416.

Gingras, Y., \& Schinckus, C. 2012. Institutionalization of Econophysics in the shadow of physics. Journal of the History of Economic Thought 34, 109 - 130. 
Hansen, L. P., \& Heckman, J. J. 1996. The Empirical Foundations of Calibration. The Journal of Economic Perspectives (1986-1998) 10, 87.

Harrison, J. M., \& Kreps, D. M. 1979. Martingales and arbitrage in multiperiod securities markets. Journal of Economic Theory 20, 381-408.

Harrison, J. M., \& Pliska, S. R. 1981. Martingales and Stochastic Integrals in the Theory of Continuous Trading. Stochastic Processes and their Applications 11, 215-260.

Hoover, K. D. 1995. Facts and artifacts: Calibration and the empirical assessmen. Oxford Economic Papers 47, 24.

Hughes, R. I. G. 1999. The Ising model, computer simulation, and universal physics. in M. S. Morgan \& M. Morrison (Eds.), Models as Mediators: Perspectives on Natural and Social Science, Cambridge University Press, Cambridge, pp. 97145.

Ingrao, B., \& Giorgio, I. 1990. The invisible hand: Economic equilibrium in the history of science MIT Press, London.

Israel, G. 1996. La mathématisation du réel. Essai sur la modélisation mathématique. Le Seuil, Paris.

Jovanovic, F. 2006a. Economic instruments and theory in the construction of Henri Lefèvre's "science of the stock market". in G. Poitras (Ed.), Pioneers of Financial Economics, vol. 1, Vol. 1, Edward Elgar, Cheltenham, pp. 169-190.

Jovanovic, F. 2006b. A nineteenth-century random walk: Jules Regnault and the origins of scientific financial economics. in G. Poitras (Ed.), Pioneers of Financial Economics, vol. 1, Edward Elgar, Cheltenham, pp. 191-222.

Jovanovic, F. 2008. The Construction of the Canonical History of Financial economics. History of Political Economy 40, 213-242.

Jovanovic, F. 2010. Efficient markets theory. in R. Cont (Ed.), Encyclopedia of Quantitative Finance, John Wiley \& Sons, Chichester (UK).

Jovanovic, F., \& Schinckus, C. 2013. The History of Econophysics' Emergence: a New Approach in Modern Financial Theory. History of Political Economy 45, 443-474.

Jovanovic, F., \& Schinckus, C. 2016. Econophysics for financial economics. Oxford University Press, New York.

Keen, S. 2003. Standing on the toes of pygmies: why econophysics must be careful of the economic foundations on which it builds. Physica A 324, 108-116.

Klass, O. S., Biham, O., Levy, M., Malcai, O., \& Solomon, S. 2006. The Forbes 400 and the Pareto wealth distribution. Economics Letters 90, 90-95.

Knorr-Cetina, K. 1981. The manufacture of knowledge. Pergamen Press, Elmsford, NY.

Kreps, D. M. 1981. Arbitrage and equilibrium in economies with in nitely many commodities. Journal of Mathematical Economics 8, 15-35.

Krugman, P. 1996. Confronting the Mystery of Urban Hierarchy. Journal of the Japanese and international economies 10, 399-418.

Le Gall, P. 2002. Les Représentations du Monde et les Pensées Analogiques des Economètres. Un Siècle de Modélisation en Perspective. Revue d'Histoire des Sciences Humaines 6, 39-64.

LeBaron, B. 2001. Stochastic volatility as a simple generator of power laws and long memory. Quantitative Finance 1, 621-631.

Lesne, A. 1998. Renormalization Methods. Critical phenomena, Chaos, Fractal Structures. John Wiley \& sons, Chichester. 
Lesne, A., \& Laguës, M. 2012. Scale Invariance: From Phase Transitions to Turbulence. Springer, Berlin.

Levy, M. H. 2003. Are rich people smarter? Journal of Economic Theory 110, 42-64.

Lucas, R. E. 1976. Econometric policy evaluation: A critique. Carnegie-Rochester Conference Series on Public Policy 1, 19-46.

Lux, T. 1996. The stable paretian hypothesis and the frequency of large returns: an examination of major german stocks. Applied Financial Economics 6, 463475.

Lux, T. 2006. Financial Power Laws: Empirical Evidence, Models, and Mechanism. in C. Cioffi-Revilla (Ed.), Power Laws in the Social Sciences: Discovering Complexity and Non-Equilibrium Dynamics in the Social Universe, Cambridge University Press, Cambridge.

Lux, T. 2009. Applications of Statistical Physics in Finance and Economics. in B. Rosser (Ed.), Handbook of Research on Complexity, Edward Elgar, Cheltenham, pp. $213-258$.

Lux, T., \& Marchesi, M. 2000. Volatility clustering in financial markets: a microsimulation of interacting agents. International Journal of Theoretical and Applied Finance 3, 675-702.

Mandelbrot, B. 1957. Application of thermodynamical methods in communication theory and in econometrics. in, Institut Mathématique de l'Université de Lille.

Mandelbrot, B. 1966a. Forecasts of Future Prices, Unbiased Markets, and "Martingale" Models. Journal of Business 39, 242-255.

Mandelbrot, B. 1966b. Seminar on the Analysis of Security Prices held November $12-13,1966$ at the Graduate School of Business of the University of Chicago. in.

Mardia, K. V., \& Jupp, P. E. 2000. Directional Statistics. Wiley, Chichester.

McCauley, J. L. 2004. Dynamics of Markets : Econophysics and Finance. Cambrigde University Press, Cambrigde.

McCauley, J. L. 2006. Response to "Worrying Trends in Econophysics". Physica A $371,601-609$.

McCauley, J. L. 2009. ARCH and GARCH models vs. martingale volatility of finance market returns. International Review of Financial Analysis 18, 151-153.

McCauley, J. L., Gunaratne, G. H., \& Bassler, K. E. 2007. Martingale option pricing. Physica A 380, 351-356.

McGoun, E. G. 2003. Finance models as metaphors. International review of financial analysis 12, 421-433.

Ménard, C. 1981. La machine et le coeur. Essai sur les analogies dans le raisonnement économique. in A. Lichnerowicz, F. Perroux \& G. Gadoffre (Eds.), Analogie et connaissance, Vol. vol. 2, chapter 14, Éditions Maloine, Paris.

Merton, R. C. 1976. Option pricing when underlying stock returns are discontinuous. Journal of Financial Economics 3, 125-144.

Mirowski, P. 1989a. The Measurement without theory controversy. Oeconomia 11, 65-87.

Mirowski, P. 1989b. More heat than light : economics as social physics, physics as nature's economics. Cambridge University Press, Cambridge ; New York.

Mitchell, M. 2009. Complexity: A guided tour. Oxford University Press, New York.

Mitzenmacher, M. 2004. A brief History of Generative Models for Power Law and Lognormal Distributions. Internet Mathematics 1, 226-251. 
Mitzenmacher, M. 2005. Editorial: The Future of Power Law Research. Internet Mathematics 2, 525-534.

Nakao, H. 2000. Multi-scaling properties of truncated Levy flights. Physics Letters A 266, 282-289.

Newman, M. 2005. Power laws, Pareto distributions and Zipf's law. Contemporary Physics 46, 323-351.

Okuyama, K., Takayasu, M., \& Takayasu, H. 1999. Zipf's Law in Income distribution of Companies. Physica A 269, 125-131.

Pagan, A. R. 1996. The econometrics of financial markets. Journal of empirical finance 3, 15-102.

Pareto, V. 1897. Cours d'Economie Politique. Librairie Droz, Genève.

Potters, M., \& Bouchaud, J.-P. 2003. More statistical properties of stock order books and price impact. Physica A 324, 133-140.

Quah, D. T. 1995. Business Cycle Empirics: Calibration and Estimation. The Economic Journal 105, 1594-1596.

Rickles, D. 2008. Econophysics and the complexity of the financial markets. in J. Collier \& C. Hooker (Eds.), Handbook of the Philosophy of Science, vol. 10: Philosophy of Complex Systems, North Holland Elsevier Editions, New York.

Rimmer, R. H., \& Nolan, J. P. 2005. Stable Distributions in Mathematica. The Mathematica Journal 9, 776-789.

Samorodnitsky, G., \& Taqqu, M. 1994. Stable non-Gaussian Random Processes. Chapman and Hall, New York.

Samuelson, P. A. 1965. Proof that properly anticipated prices fluctuate randomly. Industrial Management Review 6, 41-49.

Săvoiu, G. G., \& Andronache, C. 2013. The Potential of Econophysics for the Study of Economic Processes. in G. G. Săvoiu (Ed.), Econophysics: Background and Applications in Economics, Finance, and Sociophysics, Academic Press, Oxford (UK), pp. 91-113.

Săvoiu, G. G., \& lorga-Simăn, I. 2013. Sociophysics: A New Science or a New Domain for Physicists in a Modern University? in G. G. Săvoiu (Ed.), Econophysics: Background and Applications in Economics, Finance, and Sociophysics, Academic Press, Oxford (UK), pp. 149-166.

Schabas, M. 1990. A world ruled by number: William Stanley Jevons and the rise of mathematical economics. Princeton University Press, Princeton, N.J.

Shinohara, S., \& Gunji, Y. 2001. Emergence and collapse of money through reciprocity. Physica A 117, 131-150.

Simkin, M. V., \& Roychowdhury, V. P. 2011. Re-inventing Willis. Physics Reports $502,1-35$.

Sims, C. A. 1996. Macroeconomics and methodology. The journal of economic perspectives 10, 105-120.

Sornette, D. 2006. Critical phenomena in natural sciences second edition ed. Springer, Berlin.

Sornette, D. 2014. Physics and financial economics (1776-2014): puzzles, Ising and agent-based models. Reports on progress in physics 77, 062001-062028.

Stanley, H. E., \& Plerou, V. 2001. Scaling and universality in economics: empirical results and theoretical interpretation. Quantitative Finance 1, 563-567.

Stumpf, M. P. H., \& Porter, M. A. 2012. Critical Truths About Power Laws. Science $335,665-666$. 